(C) () 2018 IEEE. Personal use of this material is permitted. Permission from IEEE must be obtained for all other uses, in any current or future media, including reprinting/republishing this material for advertising or promotional purposes, creating new collective works, for resale or redistribution to servers or lists, or reuse of any copyrighted component of this work in other works.

DOI: $0.1109 /$ INDIN.2018.8472053

Publisher version: https://ieeexplore.iee.org/document/8472053 


\title{
SGAM-based comparative study of interoperability challenges in European flexibility demonstrators: methodology and results
}

\author{
Friederich Kupzog*, Olivier Genest ${ }^{\dagger}$, Amir Ahmadifar $^{\ddagger}$, Fabien Berthome $^{\dagger}$, Marco Cupelli ${ }^{\ddagger}$, \\ Jawad Kazmi*, Milica Savic*, Antonello Monti ${ }^{\ddagger}$, \\ *AIT Austrian Institute of Technology, Vienna, Austria, \\ Email: firstname.lastname@ait.ac.at \\ ${ }^{\dagger}$ TRIALOG SA, 25 rue du Général Foy, 75008 Paris, France, \\ Email: firstname.lastame@trialog.com \\ ${ }^{\ddagger}$ RWTH Aachen University, Germany, \\ Email: aahmadifar@eonerc.rwth-aachen.de
}

\begin{abstract}
In the European demonstration Project InterFLEX, five different demonstration sites are realized with a focus on flexibility services from energy generation and demand. The use of flexibility for grid and generation-supply balance management is a key factor in renewable-based electricity systems. However, the way such flexibility services are activated from an ICT perspective requires harmonization across Europe. As s step on this way, this paper proposes a methodology based on the Smart Grid Architecture Model (SGAM) to study cross-demonstrator ICT interoperability and show its application in the InterFLEX context. This is a multi-player environment with Distribution System Operators (DSOs), aggregators, Electric Vehicle (EV) charging operators and other actors, providing a representative overview of contemporary flexibility use and implementation options. In particular, a SGAM clustering approach is set up in order to group devices and actors within common entities. The results of this analysis show that the use-cases and interfaces are comparable between the demos, however the chosen solutions and protocols are manifold and very demonstrator-specific. Future candidates for future selection of interfaces and protocols from an InterFLEX point of view are discussed.
\end{abstract}

Index Terms-interoperability, flexibility, smart grid, distribution system operator

\section{INTRODUCTION}

The use of flexibility for grid and generation-supply balance management is a key factor in renewable-based electricity systems. However, the way such flexibility services are activated from an ICT perspective requires harmonization across Europe, as there are multiple different approaches currently under evaluation. In a future system, flexibility providers should be technically able to provide flexibility in the same or very similar way in all European member states. This is the motivation for an interoperability study within the InterFLEX European project.

\section{A. Overview of the InterFLEX project and its objectives}

The InterFLEX project [1] is a response to the Horizon 2020 Call. It explores pathways to adapt and modernize the electric distribution system in line with the objectives of the 2020 and 2030 climate-energy packages of the European
Commission [2]. Six demonstration projects are conducted in five EU Member States (Czech Republic, France, Germany, the Netherlands and Sweden) in order to provide deep insights into the market and development potential of the orientations that were given by the call for proposals, i.e., demand-response, smart grid, storage and energy system integration.

\section{B. Focus of the study described in this paper}

Cross-cutting issues are of high relevance when trying to overcome the barriers for sustainable commercial models of flexibility service provision.Therefore, the Horizon 2020 program explicitly requires performance of a detailed analysis of current regulations, standards and interoperability/interfaces issues applying to their case, more specifically in connection to ongoing work in the Smart Grid Task Force and its Experts Groups in the field of Standardisation (e.g. CEN-CLC-ETSI $\mathrm{M} / 490$ ) [3]. In particular, interoperability and standards are key enablers to allow the replicability of the project results, by ensuring a harmonized solution between EU countries. The work detailed in this paper replies to these expectations by assessing the interoperability of the demonstrated solutions, at several layers, and based on the Smart Grid Architecture Model. Individual ICT architectures of the five InterFLEX European partners allow for a systematic deduction of general and specific interoperability challenges in ICT solutions for flexibility provision, as the scope of the topics under study from different InterFLEX demonstrators is substantially wide.

\section{Related work}

This study is based on SGAM that defines a threedimensional cube, i.e. a modeling space in which smart grid applications can be clearly positioned with all their subcomponents and sub-systems, as well as associated interfaces. Different implementation variants can be easily compared. Integration with software-engineering tool chains for tracking of requirements and model-based system design is also possible [4]. Since its publication, the SGAM model together with the 
use-case methodology has already been applied to many other domains, e.g. Ambient Assisted Living or Industrie 4.0 [5].

The SGAM model is also the basis for a use-case-based methodology (IEC 62559) to design and document Smart Grid applications in a standardized way [6]. This can be seen as state of the art approach for model-based Smart Grid application design. However, although the IEC 62559 method is a formalized approach, automatic code generation and architecture evaluation is still strongly relying on the user's ability to correctly model the relevant system components in a way that supports such meta-level analysis. More formalized approaches are e.g. using domain-specific modeling languages such as proposed in [7].

Further SGAM-based analysis approaches focus on cyber security. In [8], a methodology for designing secure smart grid architectures is discussed where a graph-based deduction of security requirements for components and subsystems is possible based on SGAM models of the overall architecture.

\section{Scientific approach for the study}

In order to analyze interoperability and interchangeability among the InterFLEX demonstrators, it is essential to identify relevant interfaces, preferably implemented in more than one instance. In an initial assessment together with responsible representatives of each InterFLEX demonstrator, a number of relevant interoperability issues have been identified qualitatively. This activity has shown that inter-domain communication (e.g. from the Distribution domain to the Customer Premises) should be in the main focus, while intra-domain links are mostly based on available solutions or are not subject to interoperability as much as the inter-domain links. Further analysis is based on detailed documentation of use-cases and respective ICT architectures developed within the individual demonstrators, where the input material is harmonized in SGAM notation (see Figure 1 for an example). This analytic process is described in details in the Section IV.

The conducted study uses input material that can be modeled as set of graphs (one per use-case). Links where actors communicate (linking SGAM domains) are selected, and graphs are merged. This results in a set of selected crossdomain links of interest for interoperability. This methodology is described in detail is section 3 .

\section{THE INTERFLEX APPLICATION AREAS}

Five DSOs, CEZ distribuce, ENEDIS, EON, Enexis, and Avacon conduct six demonstration projects in five EU Member States, i.e., Czech Republic, France, Sweden, the Netherlands, and Germany. These demonstrations run 18 separate usecases validating the role of DSOs in increasing the local energy system flexibility. In association with power system experts, retailers, and manufacturers, these use-cases provide deep insights into one or several orientations focused on energy storage technologies (electricity, heating, and cooling), demand response considering two coupling of networks (electricity and gas, electricity, and heating/cooling), smart grid automation, and the integration of grid users owning electric

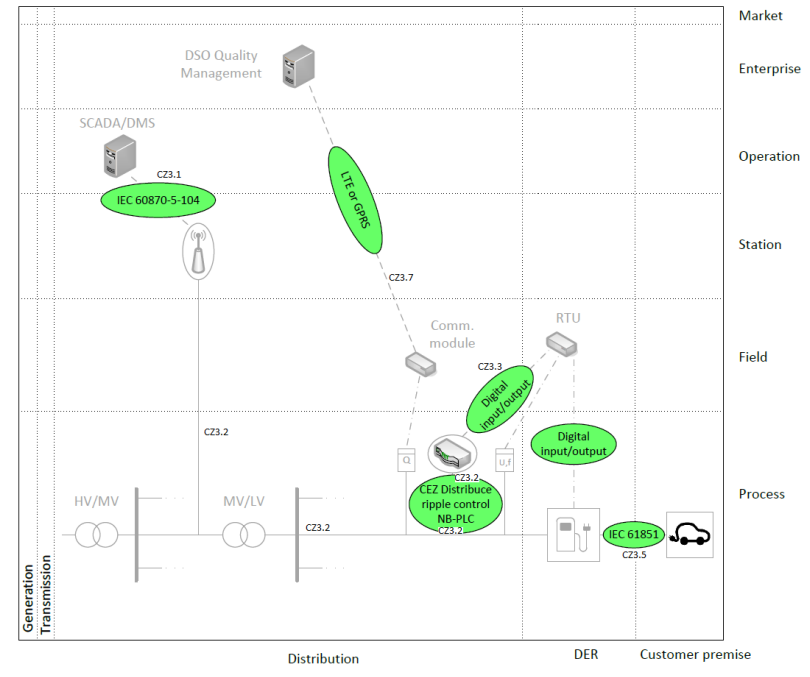

Fig. 1. Communication layer modeled with SGAM for one of the use-cases used as input.

vehicles. These use-cases can be categorized in three groups. Three use-cases in Sweden and Czech Republic focus at the enhancement of the distribution network flexibility itself. Ten use-cases in Czech Republic, France, The Netherlands, and Sweden address the network automation enhancement as well as the increased level of aggregation where both distributed generation and controllable loads bring about local energy system flexibilities. Five use-cases in France, Germany, and Sweden validate the role of IT solutions for faster automation of distribution systems making use of either local single or aggregated flexibilities.

The objectives of different use-cases are briefly described as follows.

\section{A. German use-cases}

Avacon develops a prototype Smart Grid HUB in Northern Germany which can work with any smart meter infrastructure while complying with EU recommendations. This HUB is linked with Avacon SCADA system and optimizes the interactions between different players. In this demonstration, 200 real customers are involved in three use-cases, DE.1, DE.2, and DE.3 with respective focuses on feed-in management, ancillary service provision, and demand response. In DE.1, 'Controlling small RES units', smart grid HUB decides which RES units should be controlled. In DE.2, 'Ancillary services provided by generation, consumption and storage devices', the signals coming from the aggregation of generation, consumption, and storage devices are coordinated with other signals such as curtailment and demand response signals. In DE.3, 'Distributed sources of flexibility within the distribution grid', demand response signals coming from different market participants are aggregated in the Smart Grid Hub according to the least intervention possible. Battery storage and cross-sectional technologies such as E-mobility and electrical heating systems provide flexibility for the distribution grid. 


\section{B. Czech Republic use-cases}

CEZ Distribuce, in use-case CZ.1, 'Increase the Distribution Energy Resource (DER) hosting capacity of LV distribution networks by combining smart PV inverter functions', has in view specific grid code modifications to allow an increase in DER hosting capacity within LV distribution grid using the new smart PV inverter functions, i.e., $\mathrm{Q}(\mathrm{U})$ and $\mathrm{P}(\mathrm{U})$. CZ.2, 'Increase the DER hosting capacity in MV distribution network by volt-var control', integrates the selected DER connected to MV networks into volt-var control system. CZ.3, 'Smart EV charging' and CZ.4, 'Smart energy storage' focus on increasing the distribution grid flexibility and resilience using respectively smart EV charging and home energy storage to stabilize the grid under emergency conditions, i.e., frequency and voltage deviations.

\section{Dutch use-cases}

Enexis aims at addressing similar challenges as CEZ Distribuce by providing merit orders for flexibility coming from local generation and consumption and where load areas may vary dynamically. Three use-cases have been defined in this regard as follows: NL.1, 'Enabling ancillary services, congestion management, and voltage support for PV integration using centralized, grid-connected storage systems which improve grid observability of prosumers, while promoting batteries in a multi-service approach', NL.2, 'Enabling the optimal activation of all available local flexibilities, using interactions between the DSO and the Charge Point Operator (CSO) in the role of aggregator using the local installed EVSEs for congestion management and voltage control', and NL.3, 'Validating technically, economically and contractually the usability of an integrated flex market based on a combination of static battery storage and EV'. This demosntrator is strongly focussing on an flexibility aggregator approach and the interplay between DSO and flexibility platforms.

\section{Swedish use-cases}

E.ON as a DSO and heat network operator addresses the enhancement of the distribution system flexibility in two different urban and rural demonstrations. The urban demo 4a implemented in Malmö includes use-cases SE.1, 'Use of DSR operation by exploiting the interaction with different energy carriers, such as district heating and district cooling' and SE.2, 'Optimal use of a large heat pump asset providing the district heating grid with heat and electricity flexibility for grid management purposes'. SE.1 uses district heating and cooling grids to create flexibility within the electricity distribution. SE.2 focuses on the deployment of heat pumps to increase energy flexibility in the district heating grid. Demo $4 \mathrm{~b}$ implemented in the rural area of Simris in Southern Sweden is a microgrid demonstration with the capability of going islanded and be powered by $100 \%$ of renewable sources. This demonstration will use its installed assets to enable the execution of three use-cases. Use-case SE.3, 'Technical management of a grid-connected local energy system that can run in an islanded mode with $100 \%$ of renewable generation' manages a Local Energy System (LES) with 100\% renewables and aims to transform passive customers into active ones. Use-case SE.4, 'Microgrid customer flexibility facilitated by a peer-to-peer market platform and enabled by Demand Side Response programs', develops a demand side response program for customer participation within the LES. Use-caseSE.5, 'Increased ability to observe and steer the operations of a microgrid in response to distribution network constraints', with the aid of machine learning algorithms improves the management of district heating and electricity grids.

\section{E. French use-cases}

The Enedis demonstrator is called Nice Smart Valley and is located in the South of France. Use-case FR.1, 'As an alternative to grid reconfiguration, DSOs can provide a continuous supply of electrical energy to industrial customers, thanks to electricity storage assets operated by the DSO or aggregators' will include emergency islanding (fault on the MV distribution grid), planed islanding (for maintenance works on the MV distribution grid), on-demand islanding (when regional control room needs to decrease load on its network), and preventive islanding (when voltage reaches critic level). This use-case uses storage system and aggregators. Usecase FR.2,'DSOs could integrate large scale storage volume and enable multi-service electricity storage operated by storage aggregators or DSO, with a focus on PV integration' is mainly focused on a multiservice approach for centralized storage systems that should be more efficient and visible for the DSO (data analysis can be easily done) and easier to manage than PV and smart inverters installed at customer premises. Use-case FR.3,'Innovative flexibilities can be aggregated and bid either on a local DSO mechanism to reduce local grid constraints or on national market to ensure global stability' aims to create and manage a local flexibility mechanism. It will involve electric vehicles, hot water storage tanks, energy storage system, hybrid boilers (gas/electric) and combined heat / power system. This use-case will use a forecast system to manage the flexibility.

In Table I, the overview of different use-cases can be found.

\section{SGAM-BASED APPROACH FOR INTEROPERABILITY ANALYSIS}

The analysis is based on detailed documentation of usecases and respective ICT architectures from the individual demonstrators. In order to identify similar interfaces as well as chosen ICT implementations, the input material is harmonised in SGAM notation. For this work, the component, communication and information layer are most relevant, while the function and business layer is only required as a reference to the implemented functionality and use-case. This results in a set of graphs (one per use-case) with the nodes representing devices or logical actors and edges representing logical or physical communication relations with associated protocols and data models. 
TABLE I

OVERVIEW OF INTERFLEX USE-CASES

\begin{tabular}{ll}
\hline Topic & Use-case \\
\hline Smart charging of EVs & CZ.3-4, NL.1-3 \\
Integration with heat network and storage to increase local system fleixibility & SE.1-2, FR.3 \\
Data exchange and control signals based on smart metering & DE.1-3 \\
Increase of DER hosting capacity & CZ.1-2 \\
Microgrid and islanding & FR.1, SE.3-5 \\
Multi-service business models for local energy storage & FR.2 \\
Demand response & DE.3, NL.3, FR.3, SE.3-4
\end{tabular}

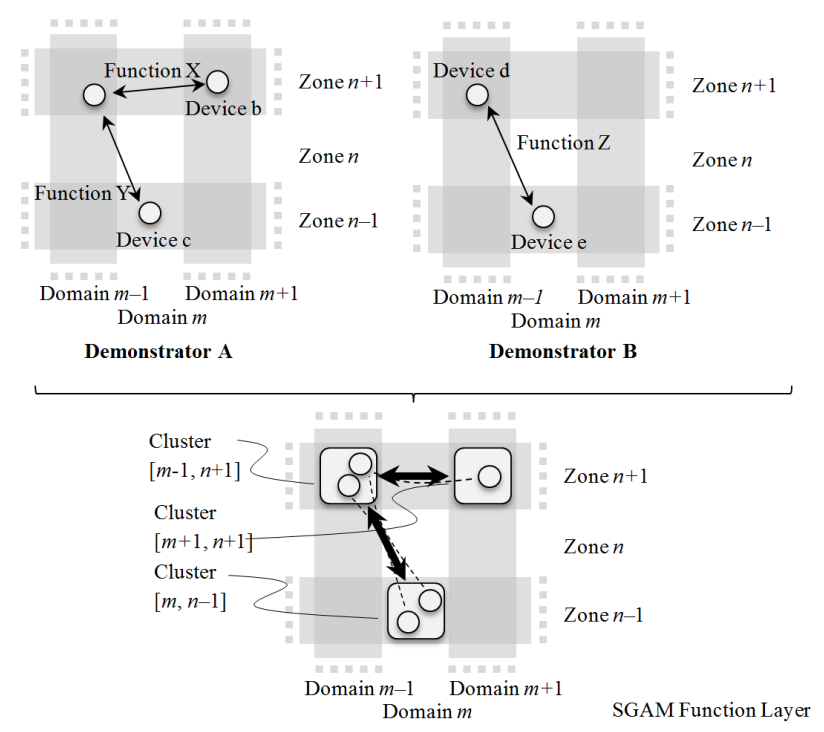

Fig. 2. Approach for clustering devices and logical actors from different demonstrators and defining bundled communication links (bold arrows)

\section{A. SGAM clustering applied to InterFLEX use-cases}

In InterFLEX, the level of devices, subsystems and systems shown in the individual architectures differs from demonstrator to demonstrator. It is therefore useful to cluster devices and logical actors across different demonstrators in larger entities in order to achieve coherent subsystem representations. For the development of the methodology, this approach proved to be effective. This approach is also supported by the fact that inter-domain links can be regarded as more relevant than intradomain communications. As shown in Figure 2, the clustering of logical actors and devices with one SGAM cell forces all individual communication relations between devices in different cells into one single bundled communication interface that supports multiple functions, potentially multiple protocols and data models, and in extreme cases even use different physical links.

\section{B. Orthogonal decomposition of diagonal links}

A closer look at the example in Figure 3 reveals that the shown configuration of a diagonal link between two different zones and two different domains is actually an abstraction done on the function layer. On the communication layer, the technical realization of such a diagonal link is most likely an orthogonal arrangement with an intermediate actor.

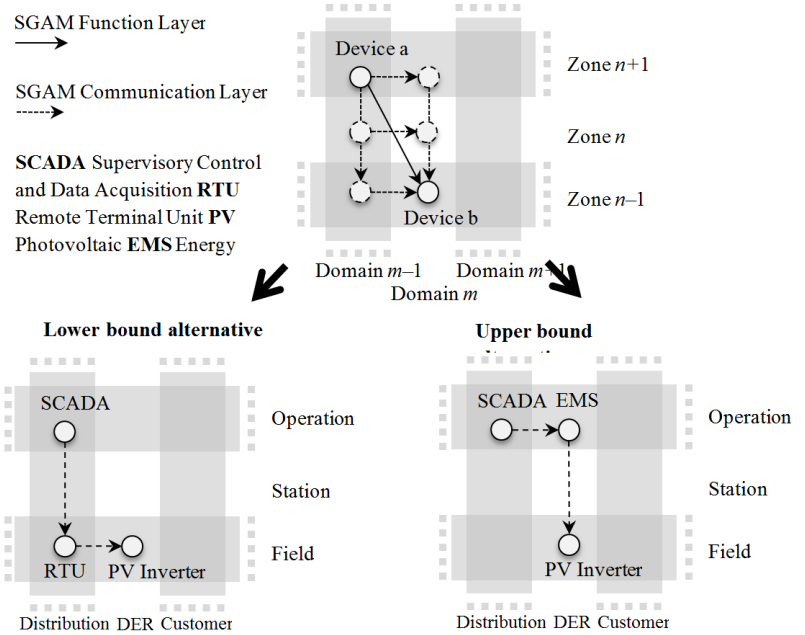

Fig. 3. The realization of a cross-domain and cross-zone communication link splits up into a series of orthogonal links.

This can be seen in an example shown in Figure 3. This SGAM diagram illustrates a PV inverter in the DER domain (Field zone) remotely monitored by the Distribution System Operators (DSOs) SCADA system. In this case, there are two principle methods to communicate between the SCADA and PV inverter.

The DSO could either employ its own Remote Terminal Unit (RTU) in the Field zone from where a monitoring interface is provided to the PV system (left side of the Figure 3). Alternatively, the DSOs SCADA system can be interfaced with the PV operational system, i.e. Energy Management System (EMS) in the Operation zone and from there the PV operator interfaces the Field device (right side of the Figure 3).

Most inter-domain connections start and end in the same SGAM zone. While this is not a strict technical necessity, there are however a number of reasons for this way of implementing cross-domain links.

1) When the system scales (e.g. DSO requires to contract more individual flexibilities), the associated engineering effort and scaling costs should be handled by the domain where the scaling occurs (in the example: customer domain). In a diagonal link scenario, handling scalability is pushed to the domain that has no direct influence on the number of devices in the scaling domain (in the example: Distribution Domain).

2) A cross-domain link has cybersecurity implications as it 
is a potentially more prominent target for cyber attacks. Definition of security measures should be based on an agreement among both domains (e.g. Distribution and Customer Premises). Connecting not only two domains but also different zones with such a link would result in a situation where one domain (e.g. Distribution) has to deal with security issues of a lower zone in another domain (e.g. customer domain field equipment), which is often undesired.

Theoretically, the horizontal link can also be made on an intermediate zone. However, this implies that not only a single but even two intermediate devices are required (or have to be upgraded) to facilitate the link. Consequently, this solution is typically not chosen for cost implications.

\section{INTEROPERABILITY AND INTERCHANGEBILITY} ANALYSIS

Within the project, a wide range of different technical and organisational constellations of flexibility exploitation is realised for the efficient operation of (electric, partly also gas or thermal) energy systems. Therefore, interoperability is critical for numerous interfaces.

In the current section, the interfaces are analysed from Interoperability and Interchangeability perspective. Due to project-wise constraints, the analysis focuses on five of the demonstrators, covering 15 of the 18 use-cases of the project.

\section{A. Criticality from the use-case perspective}

Since InterFLEX use-ases are of a broad scope (islanding, electric vehicle, demand response, energy storage, grid automation and cross energy carrier synergies), a set of generalized players in the context of flexibility can be identified, representing widely known concepts:

1) DSO, making direct or indirect (via aggregators or microgrid interfaces) use of flexibility to operate its infrastructure more efficiently. Voltage control, congestion management and increased reliability of supply are the main motivators for this.

2) Microgrid controllers that take over the energy management in a locally distinct area, with the ability to independently operate a microgrid in an off-grid mode under certain circumstances.

3) Flexibilities, which can be of very different nature, including customer-side or grid batteries, electric mobility, small-scale (boilers, domestic heat pumps, smart appliances) or large-scale power-to-heat applications.

Communication between these generalized players is fully meshed, when taking together all InterFLEX use-ases. It is dofficult to highlight a set of critical interfaces in terms of interoperability and interchangeability from this perspective due to the diversity in use-ases. It can, however, be observed that there are certain architectural patterns requiring interoperability on respective but different interfaces. We, therefore, add another viewpoint to the interoperability analysis, which is the criticality from the chosen solution and the occurrence perspective.

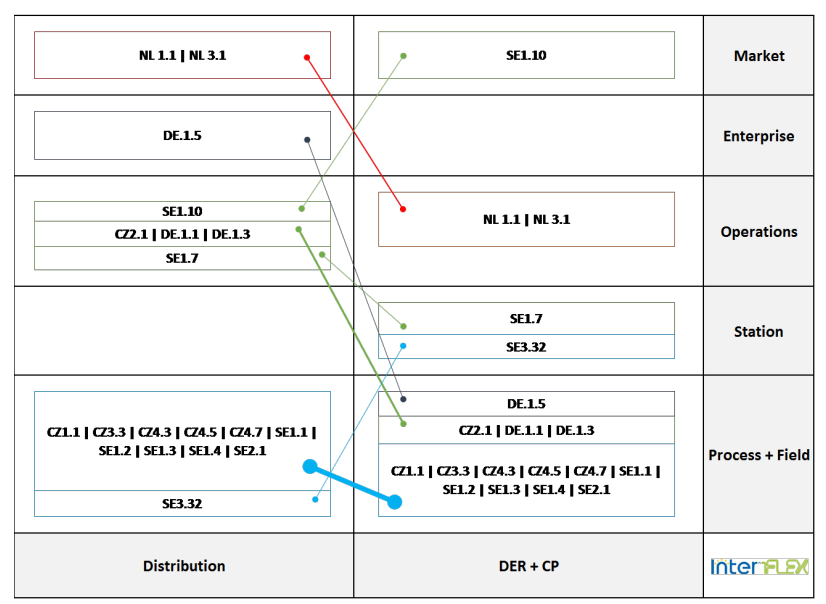

Fig. 4. Use-case mapping for interfaces. Text in cells refers to use-case identifiers. The bolder the link, the more interfaces are clustered in the one connection line.

\section{B. Criticality of the chosen solution}

In the beginning of the InterFLEX project, workshops were arranged locally at each demonstrator site with responsible people to establish a good understanding of the use-cases and chosen architecture and solutions. In these workshops, interfaces were discussed that are critical in terms of interoperability, i.e. it was at that stage not fully clear how and based on which standards it would be the best way to achieve interoperability among the actors on both sides of the interfaces. During these workshops, for each use-case the interfaces with open interoperability issues where marked. From those, seven out of the thirteen selected interfaces are associated with devices in the customers field and process zone. The main motivation for the selection of those interfaces was that the current solution implemented in the demonstrator was chosen as a specific solution for use-case due to the lack of a harmonized approach to realise functionalities on the respective interface. Similar to the interoperability analysis from other viewpoints, the large variety in solutions selected in InterFLEX also here results in a long list of very different interfaces, where interoperability analysis needs a close look on each of those interfaces. We therefore add another viewpoint to the interoperability analysis, which is the criticality from the occurrence perspective.

\section{Criticality from the occurrence perspective}

The methodology described in section III has been used in order to compare interfaces. Also, the Distributed Energy Resources and the Customer Premises domains have been merged, because we are focusing on the link between Distribution and Flexibilities, wherever they are placed (i.e. in the DER station or in the customer premises). Only the horizontal and diagonal links have been kept, as vertical links are within the same domain and do not represent any crossdomain interoperability issue. On Figure 4 we can clearly see that the most represented interface is from Distribution domain 
/ Process + field zone to DER + Customer Premises (CP) domain / Process + field zone.

D. Selection of interfaces for interoperability and interchangeability testing

When combining the interfaces highlighted by several described perspectives, the following interfaces show the most interest:

1) Distribution/Process + Field to DER + CP/Process + Field

2) Distribution/Operation to DER + CP/Market

When putting those interface into their context within InterFLEX use-cases, we obtain the following couple of actors/devices:

1) For interface 1: a. Field gateway and storage b. Field gateway and smart appliances

2) For interface 2: a. DSO SCADA and aggregators

\section{FINDINGS AND NEXT STEPS}

The clustering analysis of ICT architectures from InterFLEX demonstrators results in a set of clustered interfaces on the SGAM function layer. This is then decomposed in lower and upper bound alternatives on the SGAM communication layer. For each communication layer clustered link, bestpractice solutions, as well as an analysis of potential gaps can be performed.

Based on the results of the above outlined study, an interoperability testing suite will be worked out and exemplary cases will be demonstrated in laboratory tests. For interoperability analysis, the main focus lies on (both lower and upper bound) horizontal interfaces since there is a cross-linking between different actors.

The interoperability will also be assessed by analysing which protocols and standards could be used to achieve the interfaces of interest (e.g. SAREF/OneM2M [9] for smart appliances, or USEF [10] for aggregators), in compliance with the use-cases requirements.

\section{CONCLUSION}

In this paper, the methodology used to compare interfaces within the demonstrators has been studied: the abstraction level of domains and zones of the SGAM model has been used. This led to eliminate the different naming of same devices among the demonstrators.

When modeling the practically realized solutions in SGAM, it became clear that for each flexibility service, there are at least two principal ICT connectivity options: either a high level interface to the customer domain and then a link down to the device, or a link down to a low level distribution device and then a low-level (e.g. on/off) connection to the flexible device. It is helpful to be aware of these options when designing a flexibility application, but also when implementing the interfaces as the options have different requirements on the interface functionality.

By selecting interfaces according to a different kind of criticality, it led to the selection of two interfaces of interest:
Distribution / (process + field) to $(\mathrm{DER}+\mathrm{CP}) /$ (process + field) and Distribution / operation to (DER + CP) / market. Those interfaces are corresponding to these actors/devices: field gateway and storage, field gateway and smart appliances and the DSO SCADA and aggregators. These interfaces in particular would require further harmonization. Communication standards for these interfaces are available, but the different options cannot be easily exchanges, as this often has effects on system architecture level.

\section{ACKNOWLEDGMENT}

The presented work is conducted with support from the European Unions Horizon 2020 research and innovation program under grant number 731289 .

\section{REFERENCES}

[1] IntrerFLEX Homepage. [Online]. Available: http://interflex-h2020.com/

[2] European Comission, "A policy framework for climate and energy in the period from 2020 to 2030." [Online]. Available: http://eur-lex.europa.eu/legalcontent/EN/TXT/PDF/?uri=CELEX:52014DC0015\&from=EN

[3] H. Englert and M. Uslar, "Europäisches architekturmodell für smart grids-methodik und anwendung der ergebnisse der arbeitsgruppe referenzarchitektur des eu normungsmandats $\mathrm{m} / 490$," in Tagungsband VDEKongress, 2012.

[4] C. Neureiter, M. Uslar, D. Engel, and G. Lastro, "A standards-based approach for domain specific modelling of smart grid system architectures," in System of Systems Engineering Conference (SoSE), 2016 11th. IEEE, 2016, pp. 1-6.

[5] M. Gottschalk, M. Uslar, and C. Delfs, The Use Case and Smart Grid Architecture Model Approach: The IEC 62559-2 Use Case Template and the SGAM Applied in Various Domains. Springer, 2017.

[6] R. Santodomingo, M. Uslar, A. Goring, M. Gottschalk, L. Nordstrom, A. Saleem, and M. Chenine, "Sgam-based methodology to analyse smart grid solutions in discern european research project," in Energy Conference (ENERGYCON), 2014 IEEE International. IEEE, 2014, pp. $751-758$.

[7] F. Andrén, M. Stifter, and T. Strasser, "Towards a semantic driven framework for smart grid applications: Model-driven development using cim, iec 61850 and iec 61499," Informatik-Spektrum, vol. 36, no. 1, pp. $58-68,2013$.

[8] M. Meisel, S. Wilker, J. Fabini, R. Annessi, T. Zseby, M. Müllner, W. Kastner, M. Litzlbauer, W. Gawlik, and C. Neureiter, "Methodical reference architecture development progress," in 5th DA-CH+ Energy Informatics Conference in conjunction with 7th Symposium on Communications for Energy Systems (ComForEn), 2016, p. 40.

[9] "ETSI TS 103264 V2.1.1 - SmartM2M; Smart Appliances; Reference Ontology and oneM2M Mapping," ETSI, Tech. Rep., 3 2017, SAREF/OneM2M.

[10] USEF - Universal Smart Energy Framework. [Online]. Available: https://www.usef.energy/ 